\title{
Primary User Channel State Tracking Based on K-Nearest Neighbors Classifier and Kalman Filter
}

\author{
Ahmed Mohammed Mikaeil, Bin Guo \& Xuemei Bai \\ School of Electronics and Information Engineering, Changchun University of Science and Technology, \\ Changchun, Jilin Province, China
}

Zhijun Wang

Jilin Engineering Research Center of RFID and Intelligent Information Processing, Jilin, Changchun, China

\begin{abstract}
Spectrum sensing use the current primary user (PU) received signal to detect PU channel state at the current time, however predicting PU channel state in the near future using previous detected channel state information can secure an efficient utilization of the unoccupied spectrum, also can help in preventing the harmful interference with the PU and solving the problem of the latency between spectrum sensing and data transmission which caused by the hardware implementation. In this paper, an algorithm Based on K-Nearest Neighbor (KNN) Classifier and Kalman Filter is proposed to detect and predict the future of the primary user channel state "the spectrum holes". The new algorithm based on training KNN classifier over a set containing some frame time slots energy test statistics along with their corresponding decisions about the presence or absence of the primary user transmission, so as to predict the decisions for new unclassified time slot, then utilize the Kalman filter to track the PU the primary user channel state by predicting the next expected spectrum holes based on the previously detected hole. Experimental results show that the proposed approach is efficient and easy to implement for detecting and tracking "predicting" the PU channel state.
\end{abstract}

KEYWORD: Channel state tracking; Channel state prediction; K-nearest neighbor classifier; Kalman Filter; Spectrum sensing time slot

\section{INTRODUCTION}

Spectrum sensing is a key function of cognitive radio to identify the available spectrum hole and prevent the harmful interference with primary users (Akyildiz \& Balakrishnan 2011). However, in order to achieve these two goals the secondary user (SU) must not only be able to detect the PU spectrum holes but must also be able to get some prior knowledge about the PU channel state in the near future simply using previous detected channel states in order to be ready to occupy or leave the channel for the PU when needed. The idea of predictive dynamic spectrum access has been introduced in (Clancy \& Walker 2006), which aims at the distribution of period length of a channel being idle. And the channel state prediction using HMM and modified Hidden Markov Model has been studied in (Peh \& Liang 2007) and in (Chen \& Qiu 2010 and 2011). Another method for tracking the state of primary users based on purely discontinuous Markov process has been studied in (Guo, B \& Yan 2013). However, all of these previous works have focused on predicting the PU channel state only .In this paper we model the whole process from detecting the primary users spectrum holes to predicting "tracking " the future of PU channel state during the sensing in one model by using KNN classifier for primary user channel state detection process and Kalman filter for predicting the primary user channel state in next time step ahead the current detected sensing time slot.

The paper is organized as follows: the system model and the method of calculating the single user threshold for training KNN classifier for the detection process "predicting PU spectrum holes" is presented in section 2. In Section 3, we introduce the method for real time prediction of the PU spectrum holes using KNN classifier, followed by the method of tracking the PU channel state " predicting the next expected spectrum hole based on the previously detected one "using Kalman filter in section 4. Simulation results are presented in section 5 . Finally, section 6 concludes this paper.

\section{SYSTEM MODEL}

In this study we consider a single user cognitive radio (CR) network utilizing $\mathrm{N}$ samples for the energy detection and $M$ sensing time slots for training the KNN classifier, as shown in Fig.1 the 


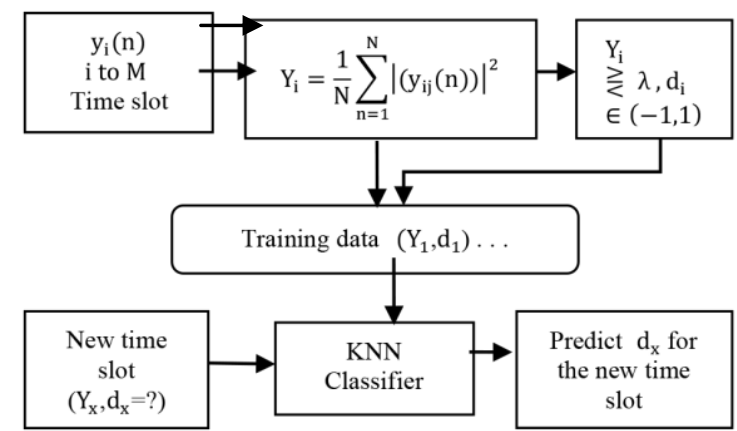

Fig. 1. The black diagram of time slot by time slot spectrum sensing based on KNN classifier

received signal of the $\mathrm{i}$-th sensing time slot contains $\mathrm{N}$ samples at the sensing node $\mathrm{y}_{\mathrm{i}}(\mathrm{n}), 1 \leq$ $\mathrm{n} \leq \mathrm{N}, 1 \leq \mathrm{i} \leq \mathrm{M}$ is given by:

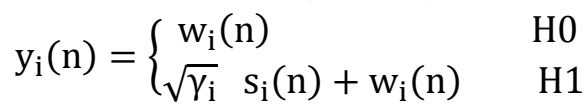

where $s_{i}(n)$ is the primary user's signal, and it is assumed to be Gaussian i.i.d random process with zero mean and variance $\sigma_{\mathrm{s}}{ }^{2}, \mathrm{w}_{\mathrm{i}}(\mathrm{n})$ is the noise, and assumed to be Gaussian i.i.d random process with zero mean and variance $\sigma_{\mathrm{u}}{ }^{2}, \gamma_{\mathrm{i}}$ the SNR at i-th time slot, $\mathrm{HO}$ and H1the hypnosis represents the PU channel state idle and occupied respectively. $s_{i}(n)$ and $w_{i}(n)$ are assumed to be independent. The energy statistic for the $i$-th sensing time slot at the sensing node $Y_{i}$ can be represented by:

$$
\mathrm{Y}_{\mathrm{i}}=\frac{1}{\mathrm{~N}} \sum_{\mathrm{n}=1}^{\mathrm{N}}\left|\left(\mathrm{y}_{\mathrm{i}}(\mathrm{n})\right)\right|^{2} \quad, 1 \leq \mathrm{i} \leq \mathrm{M}
$$

where $Y_{i}$ is a random variable whose probability density function (PDF) is chi-square distribution with $2 \mathrm{~N}$ degrees of freedom for complex value , and with $\mathrm{N}$ degrees of freedom for real value case. If we assume that the channel remains unchanged during the observation interval and that a sufficient number of samples are observed $(\mathrm{N} \geq 200)$, then $Y_{i}$ can be approximated by Gaussian distribution and the distribution of the power test $Y_{i}$ for wide band signal follows:

$$
Y_{i}= \begin{cases}\left(\sigma_{i}{ }^{2}, 2 \sigma_{i}^{4} / N\right) & H 0 \\ \left(\sigma_{i}{ }^{2}\left(1+\gamma_{i}\right), 2 \sigma_{i}^{4}\left(1+\gamma_{i}\right)^{2} / N\right. & H 1\end{cases}
$$

If we assume that the noise variance and the SNR at the node stay the same during the training process for all $\mathrm{M}$ time slot $\sigma_{\mathrm{i}}{ }^{2}=\sigma_{\mathrm{u}}{ }^{2}, \gamma_{\mathrm{i}}=\gamma_{\mathrm{u}}$, then for a chosen threshold $\lambda_{\mathrm{i}}=\lambda$ for each frame in the training set, the probability of the false alarm $\mathrm{P}_{\mathrm{f}}$ as given in (Peh, \& Liang 2007) can be written as:

$$
\begin{aligned}
\mathrm{P}_{\mathrm{f}}(\lambda)=\operatorname{Pr}\left(\mathrm{Y}_{\mathrm{i}}>\lambda \mid \mathrm{H} 0\right) & =\frac{1}{\sqrt{2 \pi \sigma_{u}}} \int_{\lambda}^{\infty} \mathrm{e}^{-\left(\lambda-\sigma_{\mathrm{u}}\right)^{2} / \sqrt{2} \sigma_{\mathrm{u}}{ }^{2}} \\
& =\mathrm{Q}\left(\left(\frac{\lambda}{\sigma_{\mathrm{u}}{ }^{2}}-1\right)\right)
\end{aligned}
$$

and the probability of detection $\mathrm{P}_{\mathrm{d}}$ is given by:

$$
\begin{aligned}
\mathrm{P}_{\mathrm{d}}(\lambda) & =\operatorname{Pr}\left(\mathrm{Y}_{\mathrm{i}}>\lambda \mid \mathrm{H} 1\right) \\
& =\mathrm{Q}\left(\left(\frac{\lambda}{\sigma_{\mathrm{u}}^{2}\left(1+\gamma_{\mathrm{u}}\right)}-1\right) \sqrt{\frac{\mathrm{N}}{2}}\right)
\end{aligned}
$$

where $Q($.$) is the complementary distribution$ function of Gaussian distribution with zero mean and unit variance. From equation (4) and for a given probability of false alarm $\mathrm{P}_{\mathrm{f}}$, the single user threshold can be written as:

$$
\lambda=\left(\sqrt{\frac{2}{N}} Q^{-1}\left(P_{f}\right)+1\right) \sigma_{u}^{2}
$$

where $Q^{-1}($.$) is the inverse of the Q($.$) function.$ The following figure illustrate the frames time slots energy statistics and the calculated detection threshed.

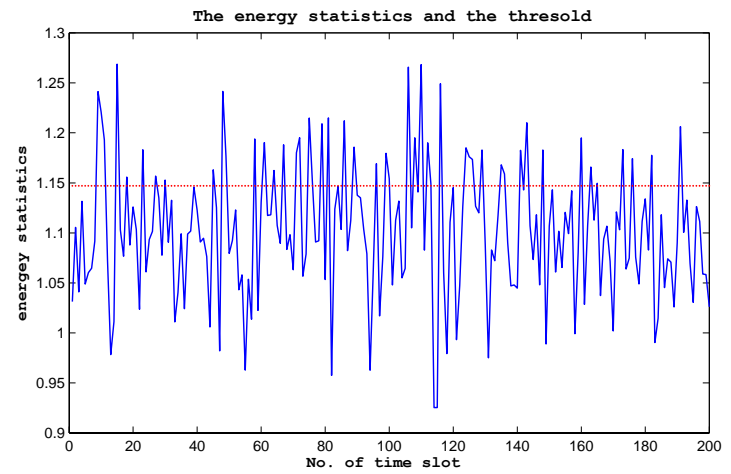

Fig. 2. Shows the frames time slots energy statistics and the calculated detection threshed

\section{REAL TIME PREDICTION OF PU CHANNEL STATE USING KNN CLASSIFIER}

The $i$-th sensing time slot energy statistic $Y_{i}$ is computed from equation (2) is compared to threshold for calculating the decision $d_{i}$ associated with the $i$ th sensing slot in the training data set which contains $\mathrm{M}$ training sensing slots as follow:

$$
\mathrm{d}_{\mathrm{i}}=\left\{\begin{array}{cl}
1 & \mathrm{Y}_{\mathrm{i}} \geq \lambda \\
-1 & \mathrm{Y}_{\mathrm{i}}<\lambda
\end{array} \quad 1 \leq \mathrm{i} \leq \mathrm{M}\right.
$$

where " -1 " represents the absence of primary user and " 1 " represents the presence of the primary user transmission on the frame. The output of equation (7) gives a set of pairs $\left(Y_{i}, d_{i}\right), i=$ $1,2 \ldots M, d_{i} \in(-1,1)$ representing the sensing time slot energy statistics and their corresponding decisions. Suppose that we would like to predict the decision "the class label" $d_{t}$ associated with a new sensing slot energy statistic $\mathrm{Y}_{\mathrm{t}}$, we can solve this classification problem using $\mathrm{KNN}$ classifier.

$\mathrm{K}$-nearest neighbors classifier use $\mathrm{K}$ nearest training points to $Y_{X}$ to predict the class label $d_{t}$ corresponding to $Y_{t}$ (Bermejo \& Cabestany 2000). For $\mathrm{K}=1$, the Euclidian distance $\mathrm{d}_{\mathrm{st}}$ between $\mathrm{Y}_{\mathrm{t}}$ and the training data points can be calculated as follow:

$$
d_{s t}(i)=\sqrt{\left(Y_{t}-Y_{i}\right)^{2}}=\left|Y_{x}-Y_{i}\right| i=1,2 \ldots M
$$

Then the new $Y_{t}$ is classified with the label $d_{t}$ $=d_{i n}$, where $d_{i n}$ is the point that has minimum Euclidian distance $d_{s t}$ to $Y_{t}$. By predicting the class label for $Y_{t}$ we get slot by time slot detection of the 
spectrum hole "the time slots with class label equal to -1 ". Our goal next in section IV is to predict the channel state or the next expected spectrum hole based on currently detected one.

\section{PU CHANNEL STATE FUTURE PREDICTION USING KALMAN FILTER}

In this section we consider tracking or predicting the primary user channel state "predicting the next expected spectrum hole based on the previously detected hole using KNN classifier in the previous section. Here, we use Kalman filter to predict the time and the state "power statistic" of the next expected spectrum hole sequence under the assumption that the appearance of the spectrum hole sequence during the time can be modeled as a dynamics movement of a simple object in onedimensional time space under the effect of a timevarying control input represented by the current detected hole sequence by $\mathrm{KNN}$ classifier. We model the tracking system state using the following equations:

$$
\begin{aligned}
& {\left[\begin{array}{l}
p_{t+1} \\
k_{t+1}
\end{array}\right]=\left[\begin{array}{ll}
\alpha_{11} & \alpha_{12} \\
\alpha_{21} & \alpha_{22}
\end{array}\right]\left[\begin{array}{l}
p_{t} \\
k_{t}
\end{array}\right]+\left[\begin{array}{l}
\beta_{1} \\
\beta_{2}
\end{array}\right] d_{t}+w_{t}} \\
& \mathrm{z}_{\mathrm{t}}=\left[\begin{array}{ll}
\delta_{1} & \delta_{2}
\end{array}\right]\left[\begin{array}{l}
\mathrm{p}_{\mathrm{t}} \\
\mathrm{k}_{\mathrm{t}}
\end{array}\right]+\mathrm{v}_{\mathrm{t}}
\end{aligned}
$$

where $\mathrm{w}_{\mathrm{t}}$ the system error source and assumed to be Gaussian noise with the distribution $\mathrm{N} \sim(0, \mathrm{Q}), \mathrm{v}_{\mathrm{t}}$ the measurement error source and assumed to be Gaussian noise with $\mathrm{N} \sim(0, \mathrm{R})$, the system state is represented $\mathrm{p}_{\mathrm{t}}$ and $\mathrm{k}_{\mathrm{t}}$ the power statistic and the number of the time slot that the next spectrum hole sequence expected to be appear during the time $t$ respectively, $d_{t}$ the control input represents the previously detected hole sequence by the KNN classifier at time $(\mathrm{t}), \mathrm{A}, \mathrm{B}$ the transition matrixes governing the dynamic movement of the hole sequences during the time, $\mathrm{C}$ the measurement matrix and $\mathrm{z}_{\mathrm{t}}$ the observed system measurement at time $\mathrm{t}$.

Our goal is to design a Kalman filter that predict $\mathrm{x}_{\mathrm{t}+1}$ from the output $\mathrm{z}_{\mathrm{t}}$ given the control inputs $\mathrm{d}_{\mathrm{t}}$ and the noisy output measurements $v_{t}$ then we can rewrite equation (9) and (10) as kalman filter state and measurement equation as in (Li \& Wang 2010) as follow:

$$
\begin{aligned}
& x_{t+1}=A x_{t}+B d_{t}+w_{t} \\
& z_{t}=C x_{t}+v_{t}
\end{aligned}
$$

Equation (11) and (12) describes a linear model at time $t+1$. As $x_{t+1}$ is not measured directly therefore, the kalman filter used the information provided by measured $\mathrm{z}_{\mathrm{t}}$ to update the unknown states $\mathrm{x}_{\mathrm{t}+1}$ by calculating the time updates and measurement updates recursively using following time and measurement updates equations:

\subsection{Time update equations}

$$
\begin{aligned}
& \hat{\mathrm{x}}_{\mathrm{t}+1 \mid \mathrm{t}}=\mathrm{A} \hat{\mathrm{x}}_{\mathrm{t} \mid \mathrm{t}}+\mathrm{Bd}_{t} \\
& \mathrm{P}_{\mathrm{t}+1 \mid \mathrm{t}}=\mathrm{AP}_{\mathrm{t} \mid \mathrm{t}} \mathrm{A}^{T}+\mathrm{B} \mathrm{QB}^{T}
\end{aligned}
$$

where

$\hat{\mathrm{x}}_{\mathrm{t} \mid \mathrm{t}}$ is the estimate of $\mathrm{x}_{\mathrm{t}}$ based on the measurement up to $\mathrm{z}_{\mathrm{t}}$.

$$
\begin{aligned}
& \mathrm{P}_{\mathrm{t} \mid \mathrm{t}}=\mathrm{E}\left(\left\{\mathrm{x}_{\mathrm{t}}-\mathrm{x}_{\mathrm{t} \mid \mathrm{t}}\right\}\left\{\mathrm{x}_{\mathrm{t}}-\mathrm{x}_{\mathrm{t} \mid \mathrm{t}}\right\}^{T}\right) \\
& \mathrm{Q}=\mathrm{E}\left(\mathrm{w}_{\mathrm{t}} \mathrm{w}_{\mathrm{t}}^{T}\right), \text { the system noise covariance }
\end{aligned}
$$

\subsection{Measurement update equations}

$$
\begin{gathered}
\hat{\mathrm{x}}_{\mathrm{t}+1 \mid \mathrm{t}+1}=\hat{\mathrm{x}}_{\mathrm{t}+1 \mid \mathrm{t}}+\mathrm{k}_{\mathrm{t}+1}\left(\mathrm{z}_{\mathrm{t}+1}-\mathrm{C} \hat{\mathrm{x}}_{\mathrm{t}+1 \mid \mathrm{t}}\right) \\
\mathrm{P}_{\mathrm{t}+1 \mid \mathrm{t}+1}=\left(I-\mathrm{k}_{\mathrm{t}+1} \mathrm{C}\right) \mathrm{P}_{\mathrm{t}+1 \mid \mathrm{t}} \\
\mathrm{K}_{\mathrm{t}+1}=\mathrm{P}_{\mathrm{t}+1 \mid \mathrm{t}} \mathrm{C}^{T}\left(\mathrm{CP}_{\mathrm{t}+1 \mid \mathrm{t}} \mathrm{C}^{T}+\mathrm{R}\right)^{-1}
\end{gathered}
$$

where

$\hat{x}_{t+1 \mid t}$ the estimate of $x_{t+1}$ given measurements up to $z_{t}$

$$
P_{t+1 \mid t}=E\left(\left\{x_{t}-x_{t+1 \mid t}\right\}\left\{x_{t}-x_{t+1 \mid t}\right\}^{T}\right)
$$

$\mathrm{R}=\mathrm{E}\left(\mathrm{v}_{\mathrm{t}} \mathrm{v}_{\mathrm{t}}^{\mathrm{T}}\right)$ is the measurement noise covariance

$\mathrm{K}_{\mathrm{t}+1}$ the Kalman gain
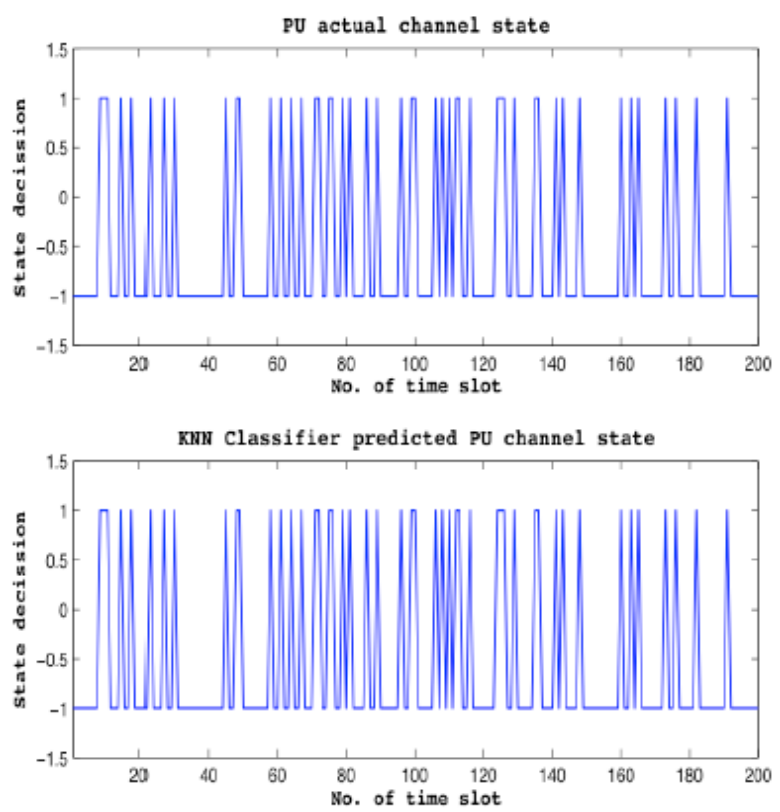

Fig. 3. Shows the performance of K-nearest neighbours (KNN) classifier used in classifying 200 time slot after training them over a set containing 200 time slot as the training examples.

\section{SIMULATION RESULTS}

Fig. 3 shows the performance of K-nearest neighbors (KNN) classifier used in classifying 200 frame time slots after training them over a set containing 200 time slots as training examples using single user threshold. For computing the single user threshold which used for the training process we assume a cognitive radio system work at SNR $\gamma_{\mathrm{u}}=-22 \mathrm{~dB}$, 
noise variances $\sigma_{\mathrm{u}}{ }^{2}=1$ and the local node decisions made after observing $\mathrm{N}=500$ samples for the energy detection under AWGN (Additive White Gaussian Noise) channel model. The figure shows that KNN classifier can classify all the 200 frames time slot correctly " achieve $100 \%$ detection rate of the spectrum holes " with only $\mathrm{M}=500$ sample used for the energy detection process. In Fig. 3 the state decision output " 1 " denotes the PU channel is in idle state while state decision output " 1 " denotes the PU channel is occupied.

Fig. 4 shows the performance of the Kalman filter in predicting the primary user channel state in one time step next the current detected channel state time step by KNN. The Kalman filter parameters used for the simulation are:
$\mathrm{A}=\left[\begin{array}{c}0.8 \\ 0\end{array}\right.$
$\left.\begin{array}{l}0.1 \\ 0.2\end{array}\right]$
$\mathrm{B}=\left[\begin{array}{l}0 \\ 1\end{array}\right] \mathrm{C}=[1$
0 ], $\mathrm{R}=1, \mathrm{Q}=1$

The first plot in Fig. 4 shows the actual PU channel state (blue line) and the tracked PU channel state (green line). In first plot the raising to the edge or falling to above -0.45 of the curve indicate the primary user transmission on channel "PU channel is occupied" while falling to the bottom or raising until less or equal to -0.45 of the curve indicate the appearance of the spectrum hole or the availability of the PU channel " PU channel is idle" for both tracking and actual PU channel state signal .The second plot compares the measurement error (dash-dot) with the estimation error (solid) which, shown that the noise level has been significantly reduced.
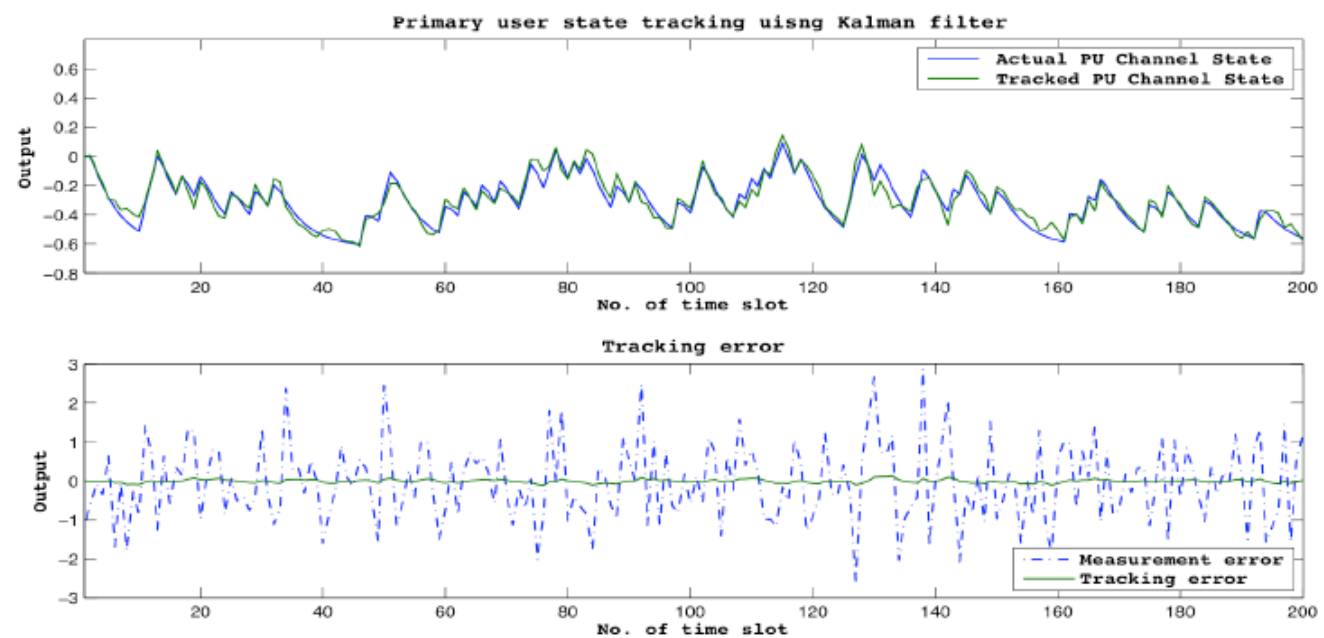

Fig. 4. Shows the performance of the Kalman filter in predicting the primary user channel state in one step next the current detected channel state time slot by KNN. The first plot shows the actual PU channel state (blue line) and the tracked channel state output (green line), and the second plot compares the measurement -error (dash-dot) with the estimation error (solid).

\section{CONCLUSION}

In this paper, we have studied the detection of the PU channel states using KNN classifier and tracking the primary user channel state "spectrum holes" using the previously detected channel state based on Kalman filter. We modeled the appearance of the spectrum hole "channel idle status" sequence during the time as a dynamics movement system and we used the Kalman filter to predict the next expected spectrum hole time step based on the currently detected spectrum hole using KNN classifier. Experimental results show that the proposed approach is efficient and easy to implement for detecting and tracking "predicting" the future of the primary user channel state.

\section{REFERENCES}

[1] Akyildiz, I. F., Lo, B. F., \& Balakrishnan, R. (2011). Cooperative spectrum sensing in cognitive radio networks: A survey. Physical Communication, 4(1), 40-62

[2] Bermejo, S., \& Cabestany, J. (2000). Adaptive soft knearest-neighbor classifiers. Pattern Recognition, 33(12),
1999-2005

[3] Clancy, T., \& Walker, B. (2006, November). Predictive dynamic spectrum access. In Proc. SDR Forum Technical Conference. 1

[4] Chen, Z., \& Qiu, R. C. (2010, March). Prediction of channel state for cognitive radio using higher-order hidden Markov model. In IEEE SoutheastCon 2010 (SoutheastCon), Proceedings of the (pp. 276-282). IEEE 4

[5] Chen, Z., Guo, N., Hu, Z., \& Qiu, R. C. (2011, March). Channel state prediction in cognitive radio, Part II: Singleuser prediction. In Proceedings of IEEE SoutheastCon ( $p p$. 50-54) 5

[6] Guo, B., Yue, X., \& Yan, X. (2013, October). Tracking the State of Primary Users Based on Purely Discontinuous Markov Process. In Cyber-Enabled Distributed Computing and Knowledge Discovery (CyberC), 2013 International Conference on (pp. 524-527). IEEE.

[7] Li, X., Wang, K., Wang, W., \& Li, Y. (2010, June). A multiple object tracking method using Kalman filter. In Information and Automation (ICIA), 2010 IEEE International Conference on (pp. 1862-1866). IEEE.

[8] Park, C. H., Kim, S. W., Lim, S. M., \& Song, M. S. (2007, December). HMM based channel status predictor for cognitive radio. In Microwave Conference, 2007. APMC 2007. Asia-Pacific (pp. 1-4). IEEE 3

[9] Peh, E., \& Liang, Y. C. (2007, March). Optimization for cooperative sensing in cognitive radio networks. In Wireless Communications and Networking Conference, 2007. WCNC 2007. IEEE (pp. 27-32). IEEE 